\title{
Calculation of Isomer Shift in Mössbauer Spectroscopy*
}

\author{
B. Fricke and J. T. Waber \\ Department of Materials Science, Northwesterm University, Evanston, Illinois 60201
}

(Received 12 November 1971)

\begin{abstract}
The approximations normally used in the calculation of the isomer shift are compared with the exact expressions using Dirac-Slater orbitals and a three-parameter Fermi-type nuclear charge distribution. The nonuniformity of the electronic density over the nuclear volume affects the results. Different choices of the nuclear surface thickness $t$ and the radius $c$ in the protonic density $\rho_{N}(r)$ also affects the isomer shift differently even though the values are chosen to yield a given value of $\Delta\left\langle r^{2}\right\rangle$. The change in the electronic charge density which is caused by the alteration of $\rho_{N}(\gamma)$ in the ground state and excited state of the nucleus is discussed using two extreme models and the possible influence on the observable isomer shift is estimated.
\end{abstract}

The Coulomb interaction energy between the charge distribution of the electrons $\rho_{e}(r)$ in an atom and the charge distribution of the protons $\rho_{N}\left(r^{\prime}\right)$ in the nucleus is given in first-order perturbation theory by the expression

$$
-e^{2} \iint \rho_{N}\left(\overrightarrow{\mathrm{r}}^{\prime}\right) \rho_{e}(\overrightarrow{\mathrm{r}}) \frac{1}{\left|\overrightarrow{\mathrm{r}}_{-}-\overrightarrow{\mathrm{r}}^{\prime}\right|} d \tau^{\prime} d \tau
$$

The usual multipole expansicn, in terms of spherical harmonics $Y_{l m}$, gives rise to the monopole interaction energy

$$
-e^{2} \iint \frac{\rho_{N}\left(r^{\prime}\right) \rho_{e}(r)}{r_{>}} d \tau^{\prime} d \tau
$$

where $r_{>}$is the larger of $r$ and $r^{\prime}$. This term with $l=0$ can be used to calculate the isomer shift whereas $l=2$ leads to the electric quadrupole interaction. Both terms can be measured in Mössbauer spectroscopy. A number of review articles have appeared ${ }^{1-6}$ on the isomer shift which deal with the increasing number of experimental results and with improved theoretical understanding. Our purpose is to discuss here the approximations used in the literature and compare them with the results obtained with the mathematically exact expressions.

The isomer shift in Mössbauer spectroscopy is nonzero when two changes occur: (a) when the nuclear charge distribution of the excited state $\rho_{N}^{*}\left(r^{\prime}\right)$ and the ground state $\rho_{N}\left(r^{\prime}\right)$ are different, and (b) when the electronic charge distribution inside the nucleus in the source $\rho_{e}^{S}(r)$ and in the absorber $\rho_{e}^{A}(r)$ are different. So the expression for the isomer shift becomes 


$$
E_{\mathrm{IS}}=-e^{2}\left(\iint \rho_{e}^{S}(r) \rho_{N}^{*}\left(r^{\prime}\right) \frac{1}{r_{>}} d \tau^{\prime} d \tau-\iint \rho_{e}^{S}(r) \rho_{N}\left(r^{\prime}\right) \frac{1}{r_{>}} d \tau^{\prime} d \tau-\iint \rho_{e}^{A}(r) \rho_{N}^{*}\left(r^{\prime}\right) \frac{1}{r_{>}} d \tau^{\prime} d \tau+\iint \rho_{\theta}^{A}(r) \rho_{N}\left(r^{\prime}\right) \frac{1}{r_{>}} d \tau^{\prime} d \tau\right)
$$

Usually two approximations are made here. First the electron charge densities in the source as well as in the absorber are assumed to be constant over the dimensions of the nucleus. This is tantamount to assuming that the nucleus is a point charge, and hence $\rho_{e}(r)$ can be replaced by $|\psi(0)|^{2}$, which is the electron density at the origin. Second, the change in the nuclear charge distribution, which can be described by the change in the second moment $\left\langle r^{2}\right\rangle$ of the nuclear charge distribution, is supposed to have no influence on the electronic charge density and vice versa. Then the expression for the isomer shift can be written in the most commonly used form

$$
\begin{aligned}
E_{\mathrm{IS}} & =\frac{2}{3} \pi Z e^{2}\left[\rho_{e}^{S}(0)-\rho_{e}^{A}(0)\right]\left(\left\langle r^{2}\right\rangle^{*}-\left\langle r^{2}\right\rangle\right) \\
& =\frac{2}{3} \pi Z e^{2} \Delta|\psi(0)|^{2} \Delta\left\langle r^{2}\right\rangle .
\end{aligned}
$$

We will discuss now the validity of both assumptions in more detail.

Recently, Shirley ${ }^{1}$ introduced a correction factor $S(Z)$ which approximately corrects nonrelativistic electron densities to the relativistic densities needed in Eq. (4). This commonly used enhancement factor $S(Z)$ can no longer be justified since relativistic Dirac-Slater ${ }^{7}$ (DS) and Dirac-Fock ${ }^{8}$ (DF) self-consistent-field (SCF) calculations are now available which include the effect of the extended nucleus. In Fig. 1, which is similar to the Fig. 1 from the paper of Kalvius, ${ }^{6}$ the relativistic electron density of ${ }_{93} \mathrm{~Np}$ (III) is compared with the nonrelativistic calculation. This shows the very strong relativistic enhancement of $\rho_{e}(r)$ near the origin, and also it becomes obvious that the assumption that the electron density is constant over the nuclear volume is not valid for large atomic numbers ever. though it is nearly valid for the nonrelativistic treatment of heavy elements. It may be very reasonable only for elements with low atomic numbers where the electrons are remote from the nucleus. A number of approximations have been used to take this effect into account. ${ }^{9-11}$ Dunlap et al. ${ }^{11}$ expand the electron charge density into a power series near the origin

$$
\rho_{e}(r)=\rho_{e}(0)\left(1-b_{1} r^{2}+b_{2} r^{4}-\cdots\right) .
$$

The presence of the additional terms leads to an isomer-shift expression of the form

$E_{\mathrm{IS}}=\frac{2}{3} \pi Z e^{2} \Delta|\psi(0)|^{2}\left(\Delta\left\langle r^{2}\right\rangle-B_{4} \Delta\left\langle r^{4}\right\rangle+B_{6} \Delta\left\langle r^{6}\right\rangle-\cdots\right)$, where the parameters $B_{4}, B_{6}, \ldots$ in front of the nuclear moments have to be adjusted. Dunlap et al. ${ }^{11}$ use as well, an even more phenomenological expression and write instead of Eq. (4),

$$
E_{\mathbf{1 S}}=\frac{2}{3} \pi Z e^{2} \Delta|\psi(0)|^{2} \Delta\left\langle r^{n}\right\rangle,
$$

where $n$ has to be adjusted.

We have compared the most commonly used approximation, Eq. (4), with the correction procedures, Eqs. (6) and (7), and with the exact calculation of the isomer shift according to Eq. (3). They have been compared for increasing values of $Z$ using a self-consistent relativistic Dirac-Slater calculation which takes into account the effect of the extended nucleus on the potential. As a model for the nucleus, we have used a three-parameter Fermi-type charge distribution

$$
\rho_{N}(r)=\frac{\rho_{N}(0)}{1+\exp \left((4 \ln 3)\left\{r\left[1-\beta Y_{20}(\theta, \varphi)\right]-c\right\} / t\right)},
$$

where $c$ is the radius at the half-density, $t$ is the surface thickness, and $\beta$ is the deformation parameter. Values for these parameters are summarized for many nuclei by Elton. ${ }^{12}$ The result is given in Fig. 2 where the two solid lines represent the percentage difference between the approximation, Eq. (4), and our exact model calculation. The two solid lines refer to two different sets of the nuclear parameters which yield the same $\Delta\left\langle r^{2}\right\rangle$. Referring

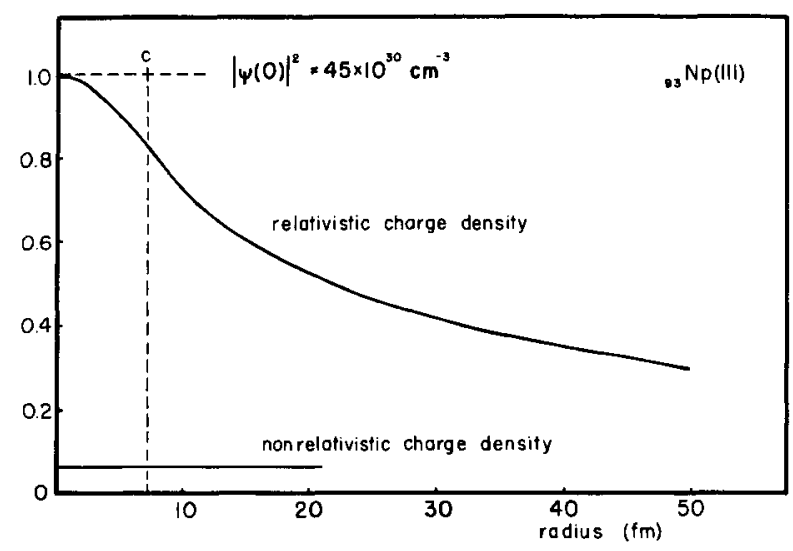

FIG. 1. Radial dependence of the electronic charge density near the nucleus for a trivalent $\mathrm{Np}$ ion. The results of both a relativistic Dirac-Slater and a nonrelativistic Hartree-Fock-Slater SCF calculations are shown. The half-density radius of ${ }^{237} \mathrm{~Np}$ is indicated by a dotted line. A very similar figure was presented by Kalvius (Ref. 6). 


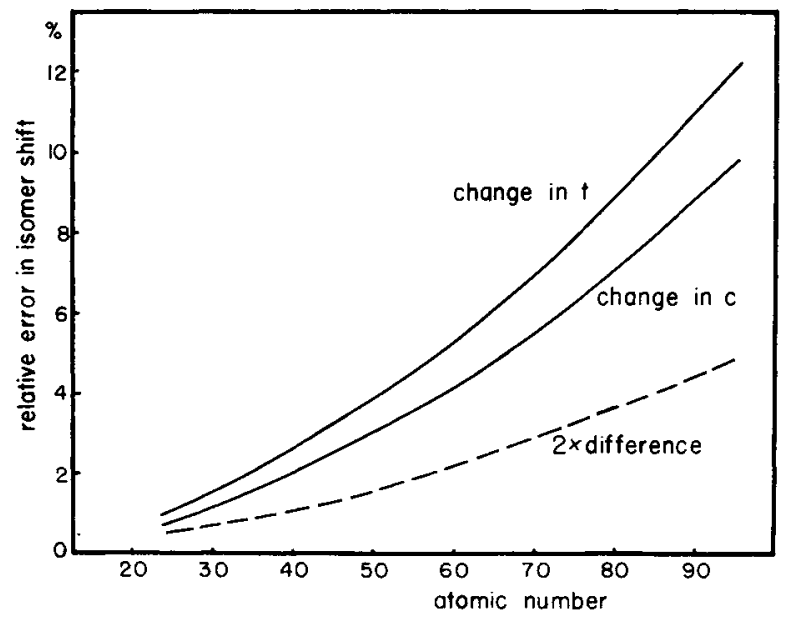

FIG. 2, The two solid lines represent the difference in percent between the isomer-shift calculation made with the normally used approximation, Eq. (4), and the exact formula, Eq. (3), and are plotted as functions of $Z$. The upper line is calculated with a change of the surface thickness $\Delta t$ and the lower with a change of the half-density radius $\Delta_{c}$ of a Fermi-type nuclear charge distribution where $\Delta t$ and $\Delta_{c}$ yield the same $\Delta\left\langle r^{2}\right\rangle$. Because of this uncertainty, all evaluations of the ratio $\rho_{1} / \rho_{2}$ (with $\left.\rho=\Delta\left\langle r^{2}\right\rangle /\left\langle r^{2}\right\rangle\right)$ of two different excited states have a principal uncertainty which is represented by the dashed line as long as one lacks more detailed information about the excited states.

to the upper curve in Fig. 2, the difference which is of the order of $11 \%$ for $\mathrm{Np}$ and $1 \%$ for Fe comes from the nonuniformity of the electron density. This large difference for heavy nuclei is too large to be neglected although the uncertainty in the determination of $|\psi(0)|^{2}$ in a range of ionic and convalent compounds may be still somewhat larger, referring to Kalvius. ${ }^{6}$ If one uses Eq. (6) to correct the nonuniformity of the electron density, the value of $B_{4}$ changes from $0.25 \times 10^{-3}$ for $\mathrm{Fe}$ to $1.1 \times 10^{-3}$ for Np.

Besides the correction due to the nonuniformity of the electron density over the nuclear volume, we get also different results for the isomer shift when one uses different changes of the nuclear charge distribution even while retaining the same value of $\Delta\left\langle r^{2}\right\rangle$. The upper solid line in Fig. 2 is calculated with a change in $t$ and the lower solid line with a change in $c$. A change in $\beta$ leads to similar values as the change in $t$ does. Because one does not yet know too much about the real change of the nuclear charge distribution between the excited state and the ground state of the nuclei, we have carried out this heuristic calculation to indicate the order of magnitude; twice the difference between the two solid lines in Fig. 2 is presented as the dashed line. For $\mathrm{Np}$, the difference is of the order of $2.5 \%$, which may look small. However, it becomes important in all analyses where the ratio $\rho_{1} / \rho_{2}$ (with
$\left.\rho=\Delta\left\langle r^{2}\right\rangle /\left\langle r^{2}\right\rangle\right)$ is evaluated for two different excited states. This ratio is given by the expression ${ }^{13}$

$$
\frac{\rho_{2}}{\rho_{1}}=\frac{E_{\gamma_{2}} F\left(Z_{1}, A_{1}\right) \Delta|\psi(0)|_{1}^{2}\left(E_{\mathrm{IS}}\right)_{2}}{E_{\gamma_{1}} F\left(Z_{2}, A_{2}\right) \Delta|\psi(0)|_{2}^{2}\left(E_{\mathrm{Is}}\right)_{1}}
$$

where $F(Z, A)=\frac{2}{5} \pi Z e^{2} R^{2}$, where $R$ is proportional to the cube root of the isotopic mass $A$, and $E_{\gamma}$ is the $\gamma$ energy of the transition. This expression is most commonly used for two excited levels in the same nucleus or in two different isotopes of the same element or between two isoelectronic pairs. The usual argument is that the electron density drops out or in the case of isoelectronic pairs the ratio of the electrondensities can be calculated with good accuracy. Figure 2 now shows that the results obtained from those analyses have a principal uncertainty of about double the difference between the two solid lines which is plotted as a dashed line in Fig. 2. This uncertainty may be adequate as long as one does not have more information about the nuclear transition and the change in nuclear charge distribution. Even with the present accuracy of measurement of the isomer shift, this uncertainty is already higher than the indicated error bars for several measurements. 9,14

This result shows that although it may be convenient to parametrize the isomer shift, neither the parameters $\left\langle\gamma^{2}\right\rangle$ and $B_{4}\left\langle r^{4}\right\rangle$ nor the parameter $\left\langle r^{n}\right\rangle$ have any large physical significance. Therefore, we propose to use the parameters $\Delta c$ and $\Delta t$ or even $\Delta \beta$ which will be a much better description of the physics involved in Mössbauer spectroscopy.

Another large difference between the relativistic and the nonrelativistic treatment is the fact that not only $n s \frac{1}{2}$ but also $n p \frac{1}{2}$ electrons contribute significantly to the total electron density near the nucleus. In the case of $\mathrm{Np}$, for example, this contribution with our DS calculation is of the order of $2 \%$, the same value as obtained by DF calculations. ${ }^{15}$ To show the even larger contribution of the $p \frac{1}{2}$ electron to the difference of the electron density near the nucleus for different electron configurations, we list in Table I the differences of electron densities for various ions and their contribution from the different shells. This shows, in the case of $\mathrm{Np}$, that neariy $20 \%$ of the change results from including the change in the $n p \frac{1}{2}$ densities. The contribution of the electrons, other than $n s \frac{1}{2}$ and $n p \frac{1}{2}$ electrons, to the isomer shift is smaller than $10^{-6}$. The differences in the electron densities in Table I show as well that the changes of the inner shells nearly cancel one another, so that the total change is given very well by the change of the outer $s \frac{1}{2}$ and $p \frac{1}{2}$ electrons only; thus, this often-used approximation is quite valid. This was already shown by Tucker et al. for gold. ${ }^{16}$ As it can be seen from Table I, 
TABLE I. The difference of the electron density $\rho_{e}(0)$ in (a. u. $)^{-3}$ between two different electron configurations for four elements separated into the contributions to the different shells from DS SCF calculations. This shows that the contributions from the inner shells nearly cancel each other and the observed change is nearly given by the change of the electron density of the outermost shell.

\begin{tabular}{|c|c|c|c|c|c|c|c|c|}
\hline \multirow{2}{*}{$\begin{array}{c}\text { Element } \\
\text { configuration } \\
\text { shell }\end{array}$} & \multicolumn{2}{|c|}{$\underset{5 s^{2}-5 s^{0}}{S n}$} & \multicolumn{2}{|c|}{$\begin{array}{c}\mathrm{Eu} \\
4 f^{6}-4 f^{7}\end{array}$} & \multicolumn{2}{|c|}{$\begin{array}{c}\mathrm{Pt} \\
5 d^{6}-5 d^{8}\end{array}$} & \multicolumn{2}{|c|}{$\begin{array}{c}\mathrm{Np} \\
5 f^{3}-5 f^{4}\end{array}$} \\
\hline & $s$ density & $p_{1 / 2}$ density & $s$ density & $p_{1 / 2}$ density & $s$ density & $p_{1 / 2}$ density & $s$ density & $p_{1 / 2}$ density \\
\hline 1 & -1.20 & $\cdots$ & -1.66 & $\cdots$ & -1.72 & $\cdots$ & -5.2 & $\cdots$ \\
\hline 2 & -0.59 & -0.02 & -3.84 & -0.21 & 0.44 & -0.10 & -11.1 & -1.7 \\
\hline 3 & -0.79 & -0.03 & -2.44 & -0.16 & 1.90 & 0.18 & -3.4 & -0.8 \\
\hline 4 & -2.17 & -0.07 & 8.85 & 0.50 & 1.76 & 0.16 & 5.3 & 0.7 \\
\hline 5 & 82.64 & & 27.48 & 1.62 & 30.28 & 3.61 & 18.3 & 3.2 \\
\hline 6 & & & & & & & 121.7 & 19.9 \\
\hline Total density & 77.89 & -0.12 & 28.39 & 1.75 & 30.95 & 3.85 & 125.6 & 21.3 \\
\hline
\end{tabular}

this statement is more correct for elements where the difference between the configurations involved are electrons with high angular momenta whose principal maxima of the wave function lie deep inside the atom, as it is in $\mathrm{Np}$ or $\mathrm{Eu}$. In the other extreme case of $\mathrm{Sn}$ where outer $s \frac{1}{2}$ valence electrons themselves are directly involved, this statement is not so true and the contribution coming from the rearranging of the inner shells has to be taken into account.

The second assumption which has been made for the derivation of Eq. (4) from (3) is that the change in electron density has no influence on the nuclear charge distribution and vice versa. The influence of the electrons on the nuclear charge is called the polarization effect, which is discussed in detail in connection with the spectroscopy of $\mu$-mesic atoms. ${ }^{17}$ Speth ${ }^{18}$ and later Mang et al. ${ }^{19}$ proved in the case of rotational transitions that there will be no observable effect in the isomer shift. For singleparticle nuclear transition a detailed calculation is still not available.

The second question concerning the influence of the different nuclear charge distribution on the electron density can be answered within our DSmodel calculation. If we assume that during the $\gamma$ transition and the rearrangement of the nucleons, the electron charge density rearranges as well to the new potential given by the new proton charge distribution, Eq. (3) has to be rewritten as

$$
\begin{aligned}
& E_{\mathrm{IS}}=-e^{2}\left(\iint \rho_{e}^{* \mathrm{~S}}(r) \rho_{N}^{*}\left(r^{\prime}\right) \frac{1}{r_{>}} d \tau^{\prime} d \tau-\iint \rho_{e}^{S}(r) \rho_{N}\left(r^{\prime}\right) \frac{1}{r_{>}} d \tau^{\prime} d \tau\right. \\
&\left.-\iint \rho_{e}^{* A}(r) \rho_{N}^{*}\left(r^{\prime}\right) \frac{1}{r_{>}} d \tau^{\prime} d \tau+\iint \rho_{e}^{A}(r) \rho_{N}\left(r^{\prime}\right) \frac{1}{r_{>}} d \tau^{\prime} d \tau\right),
\end{aligned}
$$

where the factor $\rho_{e}^{*}$ in the first and third terms refers to the electron density in the potential of the excited nucleus. The absolute change of the electron density for the two different nuclear potentials due to the ground state and the excited state is of the order of $80-100 \times 10^{26} \mathrm{~cm}^{-3}$ in the case of ${ }_{93} \mathrm{~Np}$ concerning two different choices of the set of nuclear parameters. This is much larger than the change due to chemical effects. But the observable effect is only the nonlinear part, which is only of the order of $0.2-0.4 \%$ of the isomer shift, hence is a very small contribution.

Throughout this discussion we have assumed full adiabaticity for the electrons, which means that the whole quantum-mechanical system of the nucleus plus electrons goes from the initial state (excited nucleus plus electrons in this potential) to the final state (ground state plus electrons in this potential) for the source and vice versa in the absorber. One could argue that this might not be quite true and that the time for the electrons to rearrange themselves is too short, especially when the nuclear transition is very rapid. To estimate how large this effect would be, we have assumed the other extreme, namely, that the electron density remains the same during the deexcitation of the nucleus (one would call this the sudden approximation). Then the expression for the isomer shift has to be written as

$$
E_{\mathrm{IS}}=-e^{2}\left(\iint \rho_{e}^{*} s(\gamma) \rho_{N}^{*}\left(r^{\prime}\right) \frac{1}{r_{>}} d \tau^{\prime} d \tau-\iint \rho_{e}^{* s}(r) \rho_{N}\left(r^{\prime}\right) \frac{1}{r_{>}} d \tau^{\prime} d \tau\right.
$$




$$
\left.-\iint \rho_{e}^{A}(r) \rho_{N}^{*}\left(r^{\prime}\right) \frac{1}{r_{>}} d \tau^{\prime} d \tau+\iint \rho_{e}^{A}(r) \rho_{N}\left(r^{\prime}\right) \frac{1}{r_{>}} d \tau^{\prime} d \tau\right) .
$$

For the extreme case of $\mathrm{Np}$, this would mean that an additional shift of the order of $250 \mathrm{~mm} / \mathrm{sec}$ would arise compared to $46 \mathrm{~mm} / \mathrm{sec}$ experimentally seen, whereas for the low-atomic-number element Sn the effect would be only $0.1 \mathrm{~mm} / \mathrm{sec}$ compared with the observed $5 \mathrm{~mm} / \mathrm{sec}$. This calculation shows us that this assumption is too extreme. Nevertheless, it might be possible that some of the outer electrons may not be able to follow in time. If this were true, for example, for only the two outer $6 s$ electrons in $\mathrm{Np}$, a contribution would result of about $0.9 \mathrm{~mm} / \mathrm{sec}$. To prove this adiabaticity condition and establish possible deviations therefrom, very precise measurements of Mössbauer spectra would be needed where the absorber and the source have the same chemistry or, alternately, the difference in the isomer shift could be determined from two experiments where the source and the absorber are chemically different but can be interchanged.
The result of those experiments would be not only interesting for Mössbauer spectroscopy but would be also a large support for other parts of atomic physics.

In conclusion, we may say first that for practical purposes the variation of the electronic charge over the range of the nucleus has to be taken into account and not approximated. Second, it is clear that the quantities $\Delta|\psi(0)|^{2}$ and $\Delta\left\langle r^{2}\right\rangle$ are not totally independent variables and that $\Delta\left\langle r^{2}\right\rangle$ is not always an adequate description for the change of the nuclear radius and that, at least, higher terms $\Delta\left\langle r^{n}\right\rangle$ should be included. Nevertheless, we believe that the use of $\Delta c$ and $\Delta t$ would be superior. Therefore, all results which give the ratio of the change of the mean-square radius of two excited states have a principal uncertainty of $1-5 \%$ (depending on $Z$ ). This uncertainty can be reduced when results which supply more information about the difference in the charge distribution of the ground state and the excited state become available.

\footnotetext{
*Work supported by the United States Atomic Energy Commission.

${ }^{1}$ D. A. Shirley, Rev. Mod. Phys. 36, 339 (1964).

${ }^{2}$ R. L. Mössbauer and M. J. Clauser, in Hyperfine Interactions, edited by A. J. Freeman and R. B. Frankel (Academic, New York, 1967), p. $497 \mathrm{ff}$.

${ }^{3} \mathrm{P}$. Kienle, in Hyperfine Structure and Nuclear Radiations, edited by E. Matthias and D. A. Shirley (North-Holland, Amsterdam, 1968), p. $27 \mathrm{ff}$.

${ }^{4} \mathrm{~L}$. Grodzins, in Proceedings of the Dubna Conference on Nuclear Structure, Dubna, 1968 (unpublished).

${ }^{5}$ D. A. Shirley, Ann. Rev. Phys. Chem. 20, 25 (1969).

${ }^{6}$ G. M. Kalvius, in International Conference on Hyperfine Interactions Detected by Nuclear Radiation, Rehovot, Jerusalem, Israel, 1970 (unpublished).

${ }^{7}$ D. A. Liberman, D. T. Cromer, and J. T. Waber, Comp. Phys. Commun. 2, 107 (1971); J. P. Desclaux, ibid. 1, 216 (1969).

${ }^{8}$ J. B. Mann, LASL Report (unpublished); J. P. Desclaux (unpublished).

${ }^{9} \mathrm{~T}$. K. Lees and P. A. Flinn, J. Chem. Phys. 48, 882 (1968).
}

${ }^{10}$ D. W. Hafemeister, J. Chem. Phys. 46, 1929 (1967).

${ }^{11}$ B. D. Dunlap, G. K. Shenoy, G. M. Kalvius, D. Cohen, and J. B. Mann, in Ref. 6.

${ }^{12}$ L. R. Elton, Nuclear Radii, (Oxford U. P., New York, 1968). See also, Landolt-Börnstein I, Vol. 2 (Springer-Verlag, Heidelberg, 1967).

${ }^{13}$ See, for example, S. L. Ruby and G. K. Shenoy, Phys. Rev. 186, 326 (1969).

${ }^{14}$ See, for example, E. Steichele, Z. Physik 201, 331

(1967); F. Wagner, J. Klöckner, H. J. Körner, H. Schaller, and P. Kienle, Phys. Letters 25B, 253 (1967).

${ }^{15} \mathrm{~J}$. B. Mann, in G. M. Kalvius, Proceedings of the Fourth International Conference on Plutonium and other Actinides, Santa Fe, New Mexico, 1970 (unpublished).

${ }^{16}$ T. C. Tucker, L. D. Roberts, C. W. Nestor, T. A. Carlson, and F. R. Malik, Phys. Rev. 178, 998 (1969).

${ }^{17}$ See, for example, C. S. Wu and L. Willets, Ann. Rev. Nucl. Sci. 19, 527 (1969).

${ }^{18} \mathrm{~J}$. Speth, Phys. Letters 31B, 513 (1970).

${ }^{19}$ H. J. Mang, J. Meyer, J. Speth, and W. Wild, Phys. Letters $\underline{32 \mathrm{~B}}, 321(1970)$. 